
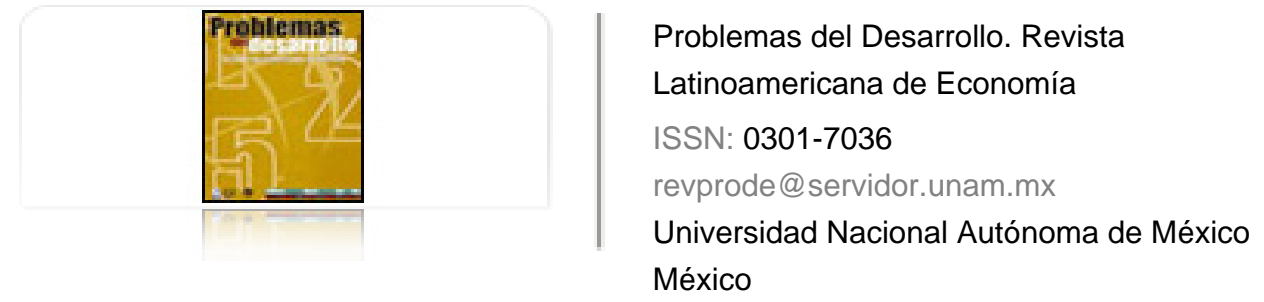

Camberos, Mario; Bracamontes, Joaquín

Marginación y políticas de desarrollo social: Un análisis regional para Sonora

Problemas del Desarrollo. Revista Latinoamericana de Economía, vol. 38, núm. 149, abril-junio, 2007, pp. 113-135

Universidad Nacional Autónoma de México

Distrito Federal, México

Disponible en: http://www.redalyc.org/articulo.oa?id=11820124006

- Cómo citar el artículo

- Número completo

- Más información del artículo

Página de la revista en redalyc.org

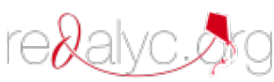

Sistema de Información Científica

Red de Revistas Científicas de América Latina, el Caribe, España y Portugal Proyecto académico sin fines de lucro, desarrollado bajo la iniciativa de acceso abierto 


\section{MARGINACIÓN Y POLÍTICAS DE DESARROLLO SOCIAL: Un ANÁlisis REGIONAL PARA SONORA}

\section{Mario Camberos* Joaquín Bracamontes**}

Fecha de recepción: 3 de octubre de 2006. Fecha de aceptación: 12 de abril de 2007.

Resumen

En nuestro país como en el resto de América Latina y el Caribe, la marginación persiste como un problema añejo del subdesarrollo. Por tal razón, nuestro objetivo es evaluar la efectividad de la política social de México a la luz de la incidencia de la marginación, definida como la carencia de uno o varios de nueve satisfactores básicos. Su medición para el presente trabajo fue con base en el índice de marginación del Consejo Nacional de Población (CONAPO), en correspondencia con Sonora y sus regiones, donde se exponen los cambios observados en las últimas décadas del siglo XX. Por otra parte, al comparar los resultados con los del CONAPO de 2000, encontramos un retroceso. Por esta razón, una de las grandes motivaciones para abordar este estudio es proporcionar herramientas para apoyar la evaluación de la misma política para otras entidades.

Palabras clave: marginación, índice de marginación, indicador, desarrollo social y región.

* Investigador titular B y profesor del Posgrado en Desarrollo Regional del Centro de Investigación en Alimentación y Desarrollo, AC, Hermosillo. Correo electrónico: mcambero@cascabel.ciad.mx

* Técnico titular A del Centro de Investigación en Alimentación y Desarrollo, AC, Hermosillo. Correo electrónico: iaco@cascabel.ciad.mx 


\section{Abstract}

In Mexico, as in the rest of Latin America and the Caribbean, marginalization persists as an old problem of underdevelopment. Therefore, our aim is to evaluate the effectiveness of social policy in Mexico in the light of the incidence of marginalization, defined as the lack of one or several of the nine basic satisfiers. The present study measures this based on the CONAPO'S IM index, in illustrative correspondence between Sonora and it regions, as well as the changes observed in the last decades of the twentieth century. Meanwhile, comparing the results with the CONAPO data for 2000, we find a reversal. For this reason, one of the important reasons for taking on this study is to provide the tools to support an evaluation of the same policy in other federal entities.

Key words: marginalization, marginalization index, indicator, social and regional development.

\section{Résumé}

Dans notre pays comme dans le reste de l'Amérique latine et dans les Caraïbes, la marginalisation persiste comme un vieux problème du sous-développement. C'est pourquoi notre objectif est d'évaluer l'efficience de la politique sociale menée au Mexique à la lumière de son incidence sur la marginalisation, définie comme l'insatisfaction d'un ou plusieurs de neuf besoins élémentaires. Pour cette étude, leur degré de satisfaction a été mesuré sur la base de l'indice de marginalisation (IM) fourni par le CONAPO (Conseil National de Population), avec illustration adjointe pour l'état de Sonora et ses régions, et par ailleurs des changements observés au cours des dernières décennies du XX'̀me siècle. D'autre part, en comparant les résultats avec ceux du CONAPO pour l'an 2000, on observe une régression. Une des principales motivations de cette étude est donc de fournir des outils pour aider à l'évaluation de la même politique pour d'autres entités géographiques.

Mots clés: marginalisation, indice de marginalisation, indicateur, progrès social et région.

\section{Resumo}

No nosso país assim como no resto da América Latina e do Caribe, a marginação persiste como um velho problema do subdesenvolvimento. Por essa razão, o nosso objetivo é avaliar a efetividade da política social do México à luz da incidência da marginação, definida como a carência de um ou vários de nove satisfatores básicos. Sua medição para o presente trabalho foi feita com base no índice IM da CONAPO, em correspondência ilustrativa de Sonora e suas regiões, além das mudanças observadas nas últimas décadas do século XX. Por outro lado, ao comparar os resultados com os da CONAPO de 2000, encontramos um retrocesso. Por esta razão, uma das grandes motivações para abordar este estudo é proporcionar ferramentas para apoiar a avaliação da mesma politica para outros estados.

Palavras-chave: marginação, indice de marginação, indicador, desenvolvimento social y região. 


\section{Introducción}

$C^{2}$

n la actualidad la marginación es un fenómeno que adquiere múltiples dimensiones geográficas en la mayoría de las regiones subdesarrolladas del mundo, como África y Asia. En América Latina, desde la década de los cincuenta se conceptuó un fenómeno similar, el de la marginalidad, referida a personas principalmente provenientes del campo, cuyas manifestaciones más visibles eran los cinturones de miseria de las grandes ciudades en sus más diversas modalidades como favelas, callampas, villas miseria, tugurios, barriadas o ciudades perdidas. ${ }^{1}$

Cabe destacar que en la última década del siglo xx, la marginación no se redujo a los cinturones de las grandes ciudades, sino que amplió su universo a localidades rurales y urbanas debido a un conjunto de factores: $a$ ) el crecimiento explosivo de la población; $b$ ) el arribo de regímenes militares en las décadas de los sesenta y setenta, que descuidaron las demandas de las mayorías; c) las crisis económicas de las dos últimas décadas multiplicaron la deuda pública y obligaron a los gobernantes a dar prioridad al cumplimiento de los compromisos financieros y a reducir el gasto social destinado a cumplir las demandas de los marginados; y $d$ ) la corrupción, que ha acompañado, casi sin excepción, tanto a regímenes militares, como a civiles latinoamericanos, multiplicó los rezagos sociales.

México, a pesar de haber desarrollado una política social activa posrevolucionaria por medio de diferentes programas, junto con la creación de las grandes instituciones de salud, reconoce que en la actualidad la marginación es un problema que flagela a numerosas localidades y municipios, particularmente de las regiones del sur y el sureste, ${ }^{2}$ en donde se conjugan, a mayores niveles, marginación y pobreza.

1 Para una crítica metodológica de los conceptos marginación y marginalidad véanse a F. Cortés, "Consideraciones sobre la marginación, la marginalidad, marginalidad económica y exclusión social”, Papeles de población, núm. 47, UAEM, 2006, pp. 71 84, así como a H. Díaz y J. Michel, Indigenismo, modernización y marginalidad, JP, México, 1981, pp. 151 168.

2 Véase al respecto, para el análisis comparativo de entidades, el trabajo de Cordera y A. Palacios, "La cohesión social en tiempos mutantes: algunos desafíos", en Economía Informa, núm. 303, México, UNAM, enero de 2003, pp. 5 21. R., cita, pp. 10 12. 
Preocupados por el problema de la marginación y la búsqueda de soluciones para resolverlo, en este trabajo nos proponemos como objetivo general la evaluación de la política social a partir de los índices de marginación (IM) y sus cambios observados en Sonora y sus regiones en las últimas décadas del siglo Xx.

Tomamos una entidad fronteriza para mostrar que la marginación la padecen, aunque con menor intensidad, pero a un nivel preocupante, otras regiones de nuestro país.

Analizamos primero el acceso a los servicios básicos y la obtención de ingreso mínimo de la población en Sonora y sus regiones, durante los periodos 1970-1990 y 1990-2000; luego, medimos cómo se tradujo en cambios en el índice de marginación. Para medirlos, utilizamos el método aplicado por CONAPO, el cual se elabora a partir de la utilización de los segundos índices de marginación IM (1990), y complementado con un método alterno que incluye regresiones lineales para estimar los IM en años que no han sido calculados, sin los cuales no sería posible conocer las tendencias de mediano y largo plazos. Lo utilizamos porque arroja resultados sólidos y es aceptado en el medio. ${ }^{3}$

\section{Concepto y medición}

\section{Definiciones}

La marginalidad, como antecedente conceptual de la marginación, en los años cincuenta adquirió relevancia como objeto de estudio, fue definida como un segmento pequeño de la población, marginada de los frutos del desarrollo económico, en comparación con los beneficios recibidos por la mayoría de los habitantes.

La marginación se define como una situación en la que viven dentro de una localidad o municipio, urbano o rural, un conjunto de individuos y familias que no satisfacen las necesidades consideradas básicas, de acuerdo con los criterios determinados por instituciones reconocidas como el Programa de las Naciones Unidas para el Desarrollo (PNUD) y el Banco Mundial.

Por población marginada también se entiende el sector de la sociedad que por causas de la organización socioeconómica y política la excluye del acceso al consumo y disfrute de bienes y servicios, y de la participación en asuntos políticos. ${ }^{4}$

3 CONAPO $\mathrm{CNA}$, Indicadores socioeconómicos e índices de marginación municipal, 1990 y 2000, México, 1993 y 2001. Véase en especial la metodología.

4 Cordera y Palacios, op. cit., p. 11. 
Si bien es cierto, como escribe Cortés (2005), son conceptos diferentes, la marginación se refiere a localidades geográficas y la marginalidad a personas, ${ }^{5}$ difieren también en que ésta no es medida; que la primera se estima en sus diferentes intensidades, a partir de las carencias de los individuos y tienen en común que aluden a poblaciones a quienes el sistema de mercado no le retribuye los derechos por satisfacer un conjunto de necesidades consideradas básicas o esenciales y quienes durante la última década del siglo XX no han recibido los beneficios esperados de la globalización.

En esa línea de razonamiento y con el objeto de hacer comparables nuestros resultados con los obtenidos por el CONAPO, en la actualidad con más reconocimiento en el tema, en este trabajo definimos la marginación como la situación en que se encuentran los individuos y las familias al interior de una localidad o municipio, que no satisfacen un conjunto de necesidades consideradas esenciales o básicas, ${ }^{6} \mathrm{y}$ que se traduce en las nueve siguientes carencias (en porcentaje): $a$ ) población analfabeta de 15 años y más (ANALF); $b$ ) población de 15 años y más sin primaria completa (SINPRI); $c$ ) de viviendas sin disponibilidad de drenaje ni excusado (SINEXDR); d) viviendas sin disponibilidad de electricidad (SINELEC); $e$ ) viviendas sin disponibilidad de agua entubada (SINAG); $f$ ) viviendas con uno y dos cuartos (HACIN); $g$ ) porcentaje de viviendas con piso de tierra (PISOTI); $h$ ) población en localidades menores de 5000; e $i$ ) población que gana hasta dos salarios mínimos de 1970.

\section{Método para medir la marginación y estimar sus tendencias}

El índice de marginación (IM) de 1990 lo construye CONAPO ${ }^{7}$ mediante una combinación lineal de los nueve indicadores arriba señalados y el método de componentes principales y un modelo del tipo:

$$
\mathrm{IM}=\mathbf{a}_{1} \operatorname{IND} 1+\mathbf{a}_{2} \operatorname{IND} 2+\mathbf{a}_{3} \operatorname{IND} 3+\ldots+\mathbf{a}_{9} \mathrm{IND} 9
$$

en el cual a representa el parámetro asociado a cada uno de los nueve indicadores $\mathrm{IND}_{\mathrm{J}}$, con a y $\mathbf{j}=1,2,3 \ldots, 9$.

Los IM de 1970, 1980 y 2000, que servirán para establecer las tendencias y las proyecciones de corto y mediano plazos, fueron calculados a partir de la siguiente metodología del propio CONAPO:

Cortés, op. cit., 2006, pp. 73 75 y 79 81.

Al respecto, véase Julio Boltvinik, Pobreza y estratificación social en México, México, INEGI, COLMEX, IIS UNAM, 1994, p. 69.

7 Para una explicación metodológica más amplia, véase CONAPO CNA, Indicadores socioeconómicos e índice de marginación municipal, México, 1993, pp. 23 35. 
- Primero. Adecuar conceptualmente las variables de los censos de 1970 y 1980 para realizar la comparación de resultados con los de 1990.

- Segundo. Estimar el segundo índice de marginación (IN2) 1990, para cada municipio, a partir de las nueve variables ( Vi ) (con i $=1,2 \ldots, 9)$. Éstas se ponderan por sus respectivos coeficientes $(\mathrm{Ci}),(\operatorname{con} \mathrm{i}=1,2, \ldots, 9)$. Tendremos, por lo tanto, que:

$$
\text { (1) } I N 2=\Sigma i \mathrm{ViCi}
$$

- Tercero. Utilizar un modelo de regresión que relaciona como variable dependiente los índices IM 1990 y los IN2 1990 como variable independiente. Así, para la región desierto se obtuvo la ecuación de regresión siguiente:

$$
\text { (2) } I M=-2.9377+0.0461(I N 2)
$$

- Cuarto. Sustituir la variable independiente por los valores IN2 de 1970 y 1980, calculados con el procedimiento seguido para 1990 y que dará como resultado los índices IM de1970 y 1980.

El IM adquiere valores positivos o negativos para las regiones y la entidad de acuerdo con la clasificación que aparece en el cuadro 1. Los valores positivos mayores indican alta marginación, en tanto que los negativos representan marginación baja. Por no estar acotado el índice entre cero y uno, se desconoce la proporción de la población que se encuentra en marginación y, por tanto, tampoco el porcentaje de cambio que sufrió con el tiempo.

Una opción sería la reescalación en el intervalo [0,1]. Hay dos problemas. a) Los intervalos no son simétricos y b) Se perdería la clasificación que califica la intensidad de la marginación.

Cuadro 1

Índices de marginación

\begin{tabular}{ll}
\hline Estrato & Rango \\
Muy baja & si su IM está en el intervalo [-2.67812, -1.58950] \\
Baja & si su IM está en el intervalo [-1.58950, -0.50461] \\
Media & si su IM está en el intervalo [-0.50461, 0.04150] \\
Alta & si su IM está en el intervalo [0.04150, 1.13059] \\
Muy alta & si su IM está en el intervalo [1.13059 2.76549] \\
\hline
\end{tabular}

Fuente: CONAPO-CNA, Índice de marginación municipal, 1993, p. 33 
No obstante, al comparar los valores del IM de un periodo a otro, podemos conocer si la marginación disminuyó, aumentó o se mantuvo constante, de acuerdo con el rango en que se ubica.

\section{La política social y los programas contra la marginación}

La política social, ahora de desarrollo social, se inscribe en el terreno de las políticas públicas. En México, la política social posrevolucionaria se inscribió en la misma Constitución de 1917, la cual se reducía inicialmente a la asistencia social, y después dio un gran salto con la creación de las instituciones sociales como el Instituto Mexicano del Seguro Social y el Instituto para la Seguridad Social de los Trabajadores del Estado. Esas políticas no tenían medidas las dimensiones de la alimentación, educación, y vivienda; no atendían al desarrollo de las capacidades básicas ${ }^{8}$ como debe ser una política social integral.

Fue a partir del régimen de José López Portillo que se consideró ese aspecto integral de la política social por medio de diferentes programas, sin éxito, aunque hay que reconocer, como lo muestran diversos estudios al respecto, que centran su análisis en la marginación y que a continuación revisamos.

Uno de los más conocidos es la Geografía de la Marginación, elaborado por la Coordinación General del Plan Nacional de Zonas Deprimidas (COPLAMAR) a fines de los setenta. El estudio realizó un mapeo muy completo de la marginación tomando en cuenta cuatro dimensiones: alimentación, salud, vivienda y educación, cuyos resultados hicieron posible la aplicación del programa de atención a los grupos deprimidos en las zonas marginadas de México. Ese programa inauguró una corriente que centra el interés de la politica económica y social en la atención de los grupos de individuos, las familias de las regiones al interior de los municipios y estados, más que en el de los grandes sectores como el petróleo, el acero, las cuencas hidrológicas, la montaña, tal y como había ocurrido anteriormente.

En la década de los noventa, la política social sufrió un cambio importante, al centrar su objetivo ya no en los grupos y regiones, sino en un nivel más desagregado de las localidades y municipios. A partir de un diagnóstico que incluyó la medición de la marginación elaborado por el CONAPO y la Comisión Nacional del Agua (CNA), Indicadores socioeconómicos e Índice de marginación municipal 1990, considerando cuatro aspectos: alimentación, salud, vivienda y educación y con base en las

8 Consúltese a Trejo y Jones, Contra la pobreza, México, Cal y Arena, 1993, pp. 151 202. 
carencias de los individuos, el gobierno de Ernesto Zedillo implantó el programa Progresa. Posteriormente, se actualizó el estudio Índices de marginación 2000 por el mismo CONAPO, ${ }^{9}$ con la misma metodología, pero con la información del Censo de Población de 2000 y ha servido para el establecimiento de los programas más recientes, Oportunidades-Contigo, fincado en la nueva política social de atención a la población de las regiones y microrregiones con más alta marginación, ${ }^{10}$ integradas por localidades y municipios.

Una vez analizado el entreteje de las políticas sociales y los programas para combatir la marginación, es necesario conocer las características de la población y la economía del espacio geográfico objeto de nuestro estudio: Sonora y sus regiones.

\section{Las regiones de Sonora}

El estado de Sonora cuenta con 72 municipios, está dividido en 12 regiones (véase Mapa 1) y ocupa el segundo lugar nacional en extensión territorial, después de Chihuahua; en 2000, su población ascendió a 2,216,969 personas, ${ }^{11}$ poco más de $2.2 \%$ del total nacional. El crecimiento de su población ha perdido dinamismo, es decir, ha sido a tasas menores en las últimas décadas, coincidiendo con la tendencia observada en el nivel nacional. ${ }^{12}$ La clasificación de las regiones que realizamos (véase cuadro 2) está basada en una regionalización elaborada por la antigua Secretaría de Programación y Presupuesto.

Utilizamos el criterio de la actividad que absorbe la mayor proporción de la población económicamente activa (PEA) ocupada para la clasificación de cada una de las regiones de la forma siguiente: agrícola (A) si más de 50\% de la PEA tiene actividades del sector primario; PA si la PEA se ocupa en un porcentaje mayor en la agricultura que en cada una de los sectores restantes. Aplicamos el mismo criterio a los otros sectores y tendremos I y PI para el industrial, y s y PS para el sector servicios. En el cuadro 2 observamos que la PEA ascendió a poco más de 800 mil personas y representó $36 \%$ del total de población en 2000, lo cual indica que había una dependencia económica de 2.73 sonorenses por cada empleado. El cuadro nos indica también que entre 1990 y 2000 las regiones observaron un cambio cualitativo puesto que en 1990 en ocho de las 12 regiones predominaban las actividades

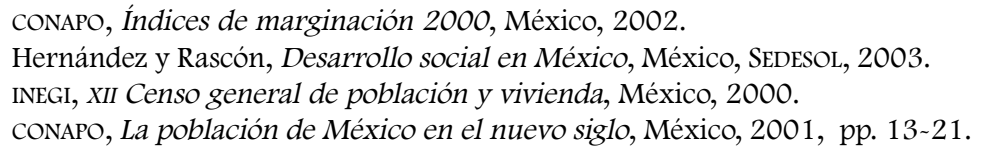


ligadas al campo; en tanto que solamente en las dos regiones fronterizas dominaban las actividades industriales, casi en su totalidad del ramo maquilador.

Para 2000 la economía de Sonora pasó a ser predominante en servicios, debido a que poco más de la mitad de la PEA, 51.3\%, se ocupaba en esa actividad, tendencia observada para México y sus entidades según el Censo de 2000.

Cabe destacar que la reconversión de las regiones no se debió precisamente a la modernización, sino que más bien fue producto del ahondamiento de la crisis de mediados de los noventa, que afectó a todas los sectores económicos, pero que se recrudeció en el campo por el llamado problema de las carteras vencidas que ya padecían los agricultores, misma que se tradujo en una mayor restric-
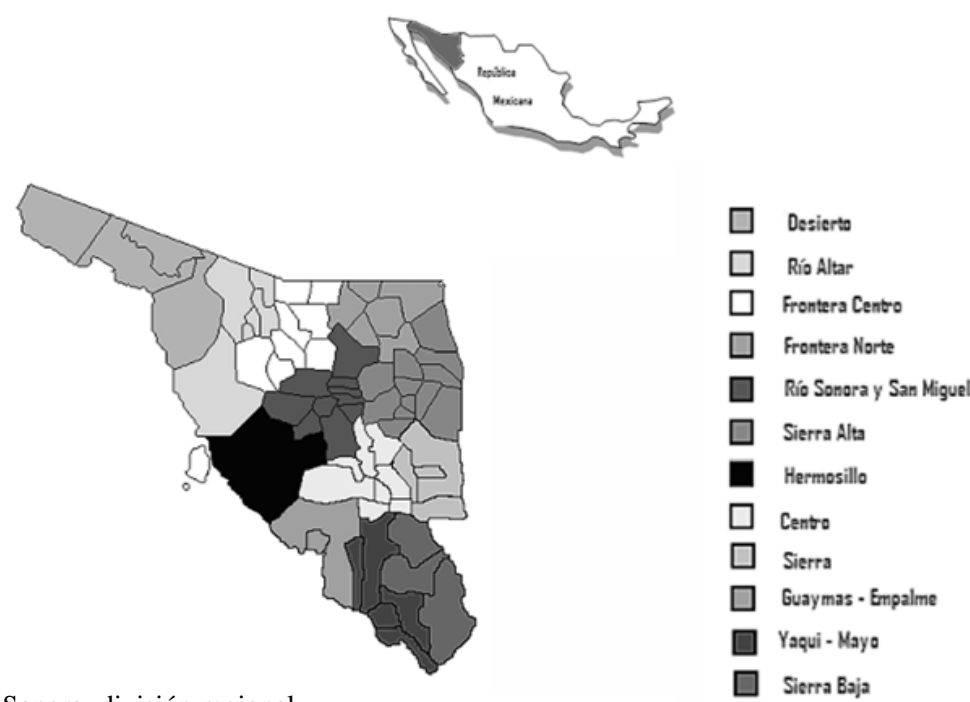

Mapa 1. Sonora, división regional

Municipios que comprende cada región. Desierto: Caborca, San Luis Río Colorado y Plutarco Elías Calles; Río Altar: Altar, Atil, Oquitoa, Pitiquito, Sáric y Tubutama; Frontera Centro: Benjamín Hill, Cucurpe, Imuris, Magdalena, Nogales, Santa Ana, Sta. Cruz y Trincheras; Frontera Norte: Agua Prieta, Bacoachi, Cananea, Fronteras, Naco y Nacozari; Río Sonora y S. Miguel: Aconchi, Arizpe, Banámichi, Baviácora, Carbó, Huépac, Opodepe, Rayón, San. Felipe, S. Miguel de Horcasitas y Ures; Sierra Alta: Bacadéhuachi, Bacerac, Bavispe, Cumpas, Divisaderos, Granados, Huachineras, Huásabas, Moctezuma, Nácori Chico, Tepache y Villa Hidalgo; Hermosillo: Hermosillo; Centro: La Colorada, Mazatán, Onavas, S. Javier, San Pedro de la Cueva, Soyopa, Suaqui Grande, Villa Pesqueira; Sierra: Arivechi, Bacanora, Sahuaripa y Yécora; Guaymas-Empalme: Guaymas y Empalme; Yaqui-Mayo: Bácum, Cajeme, Etchojoa, Huatabampo, Navojoa y San Ignacio Río Muerto; Sierra Baja: Alamos, Quiriego y Rosario Tesopaco. SPP, Gobierno de Sonora, Plan Estatal de Desarrollo, 1980-1985, Hermosillo, 1980.

Fuente: elaboración propia con base en la regionalización de gobierno de Sonora del Plan Estatal de Desarrollo, 1980-1985, Hermosillo, Son., 1980. 
ción del crédito para las actividades agropecuarias, ${ }^{13}$ al constituirse el llamado buró de crédito, razón por la cual miles de productores del campo se convirtieron en prestadores de servicios, sobre todo en la región Yaqui-Mayo, que en el periodo 1990-2000 dio el salto de una economía predominantemente agrícola a otra de servicios.

Cuadro 2

Población y PEA ocupada por regiones de Sonora, 1990 y 2000

\begin{tabular}{|c|c|c|c|c|c|c|c|}
\hline Entidad & $\begin{array}{l}\text { Población } \\
\text { total } 2000\end{array}$ & $\begin{array}{c}\text { Ocupados } \\
2000\end{array}$ & $\begin{array}{l}\text { Clasificación } \\
2000 \\
\text { en } \\
\text { má }\end{array}$ & $\begin{array}{l}\% \\
\text { de la PEA } \\
\text { ala actividad } \\
\text { ás importante } \\
1990\end{array}$ & $\begin{array}{c}\text { Ocupados } \\
1990\end{array}$ & $\begin{array}{c}\text { Clasificación } \\
1990 \\
\text { en lo } \\
\text { más }\end{array}$ & $\begin{array}{c}\% \\
\text { de la PEA } \\
\text { a actividad } \\
\text { importante } \\
1990\end{array}$ \\
\hline SONORA & $2,216,969$ & 810,424 & $\mathrm{~s}$ & 51.3 & 562386 & PS & 49.0 \\
\hline 1. Desierto & 256,957 & 97,140 & PS & 48.0 & 60,813 & PS & 27.9 \\
\hline 2. Río Altar & 21,664 & 7,826 & PS & 36.8 & 3,330 & PA & 34.7 \\
\hline 3. F. Centro & 217,801 & 84,852 & PI & 47.2 & 54,157 & PI & 38.3 \\
\hline 4. F. Norte & 123,037 & 44,137 & PS & 46.7 & 28,715 & PI & 26.7 \\
\hline $\begin{array}{l}\text { 5. Río Sonora } \\
\text { y San Miguel }\end{array}$ & 37,179 & 13,346 & $\mathrm{PA}$ & 46.0 & 10,650 & $\mathrm{PA}$ & 29.7 \\
\hline 6. Sierra Alta & 24,414 & 7,841 & $\mathrm{PA}$ & 34.2 & 7,624 & $\mathrm{PA}$ & 47.1 \\
\hline 7. Centro & 10,765 & 3,797 & $\mathrm{PA}$ & 40.4 & 3,711 & A & 50.3 \\
\hline 8. Hermosillo & 609,829 & 234,996 & $\mathrm{~S}$ & 60.5 & 146,852 & PS & 35.5 \\
\hline 9. Sierra & 14,896 & 4,617 & $\mathrm{PA}$ & 38.1 & 3,993 & A & 55.5 \\
\hline $\begin{array}{l}\text { 10. Guaymas- } \\
\text { Empalme }\end{array}$ & 180,316 & 65,264 & PS & 48.5 & 52,253 & $\mathrm{PA}$ & 26.4 \\
\hline 11. Yaqui-Мayo & 686,192 & 236,745 & $\mathrm{~s}$ & 51.8 & 176,331 & PA & 29.7 \\
\hline 12. Sierra Baja & 33,919 & 9,863 & A & 44.2 & 8,677 & A & 61.5 \\
\hline
\end{tabular}

Fuente: estimaciones propias con base en los XI y XII Censo General de Población y Vivienda 1990 y 2000, así como el Plan Estatal de Desarrollo del Estado de Sonora, 1980.

El paso de una población dominada en 1970 por las actividades ligadas al campo, que apenas rebasaba el millón de habitantes a otra caracterizada por la economía de los servicios en 2000 con más de dos millones 200 mil habitantes, debió haber sido acompañada por transformaciones en la calidad de los indicadores básicos en general para la sociedad sonorense, como ocurre con todas las sociedades

13 Mario Camberos y Jaime Yánez, "La pobreza regional en los 1990s: estrategia, medidas para combatirla y costos de financiamiento", en Economía y sociedad, año 9, núm. 13, Morelia, México, Facultad de Economía, Universidad Michoacana de San Nicolás de Hidalgo, enero junio de 2004, pp. 37 51. 
que transitan por ese camino; sin embargo, no sabemos si los logros obtenidos durante el periodo produjeron cambios proporcionales en todos los indicadores ni tampoco si las regiones avanzaron al mismo ritmo. En seguida trataremos de dar respuesta a tales interrogantes.

\section{Los indicadores de carencia y su tendencia 1970-1990}

Para establecer la tendencia de los indicadores en la entidad y las regiones, interesa tomar su valor en cada uno de los años y considerar sus diferencias porcentuales. Anotamos los indicadores de carencia para 1970 y 1990, pero aunque no aparezca el cuadro para 1980, hemos considerado los datos para estimar el IM de ese año. El análisis de los indicadores de 1970 (véase cuadro 3) muestra que Sonora registró casi 15\% de la población de 12 años y más como analfabeta; mientras que sin primaria completa fue de $63.49 \%$; sin electricidad $35.39 \%$; sin agua entubada $31.65 \%$ y viviendas con piso de tierra bajó a $37.41 \%$; sin drenaje ni excusado para quedar en $58.16 \%$; hacinamiento con $58.39 \%$ y localidades con menos de 5,000 habitantes 39.65\%; la PEA con ingreso menor a dos SM fue de $36.09 \%$. La comparación entre las regiones muestra el contraste entre las fronterizas, no agrícolas, con los porcentajes menores de carencia en todos los rubros y las tres serranas, y la del Río Sonora y San Miguel, agrícolas, observan las mayores carencias.

Cuadro 3

Sonora, indicadores de carencia regional, 1970

\begin{tabular}{lrrrrrrrrr}
\hline & ANALF & SINPRI & SINDEXDR & SINELEC & SINAG & HACIN & PISOTI & LOC5000 & INGR \\
\hline Sonora & 14.80 & 63.49 & 58.16 & 35.39 & 31.65 & 58.39 & 37.41 & 39.65 & 36.09 \\
Desierto & 13.36 & 70.48 & 63.56 & 29.36 & 27.13 & 59.32 & 25.20 & 24.57 & 27.45 \\
Río Altar & 12.89 & 71.80 & 77.90 & 59.79 & 50.20 & 49.14 & 41.39 & 100.00 & 41.89 \\
F. Centro & 10.23 & 53.95 & 46.92 & 26.76 & 24.05 & 46.54 & 21.23 & 21.80 & 30.87 \\
F. Norte & 8.05 & 54.09 & 42.21 & 26.50 & 18.30 & 47.70 & 21.66 & 27.42 & 32.41 \\
R. Sonora & 12.18 & 71.10 & 70.40 & 49.71 & 37.19 & 56.28 & 44.39 & 100.00 & 51.93 \\
y S. Miguel & & & & & & & & & \\
Sierra Alta & 11.49 & 70.47 & 80.53 & 74.47 & 43.03 & 49.41 & 58.72 & 100.00 & 50.76 \\
Centro & 13.51 & 79.68 & 77.48 & 73.88 & 49.17 & 66.49 & 53.31 & 100.00 & 44.54 \\
Hermosillo & 11.46 & 54.86 & 46.39 & 21.78 & 23.89 & 51.07 & 25.72 & 15.16 & 31.24 \\
Sierra & 18.39 & 80.43 & 87.12 & 66.23 & 54.63 & 62.54 & 66.55 & 100.00 & 44.77 \\
Guaymas- & 15.53 & 72.85 & 59.38 & 33.17 & 29.49 & 58.92 & 38.48 & 31.01 & 33.24 \\
Empalme & & & & & & & & & \\
Yaqui-Mayo & 17.98 & 59.65 & 59.79 & 36.01 & 34.66 & 65.16 & 45.92 & 42.27 & 38.55 \\
Sierra Baja & 18.19 & 65.74 & 86.34 & 83.14 & 70.47 & 82.83 & 79.26 & 100.00 & 58.02 \\
\hline
\end{tabular}

Fuente: estimaciones propias con base en CONAPO 1993 y Ix Censo General de Población y Vivienda de 1970 . 
Para 1990 (véase cuadro 4) se observaron logros en casi todos los rubros, aunque fueron sensiblemente menores en educación y en drenaje. La excepción fue el de la población con ingresos menores a dos salarios mínimos (SM), que rebasó 50\% de la PEA en la entidad, síntoma de empeoramiento económico continuo también de los hogares con empleados. Por regiones, Hermosillo registró los mayores avances en cinco de los nueve indicadores; contrario a lo ocurrido con la Sierra Baja, que mantuvo el mayor porcentaje de carencia en todos los indicadores - excepto localidades menores de 5,000 — con el agravante que ha visto empeorar su situación con el tiempo, al grado que guarda mayores diferencias con el resto de las regiones que las observadas en 1970.

Cuadro 4

Sonora, indicadores de carencia regional, 1990

\begin{tabular}{lrrrrrrrrr}
\hline & ANALF & SINPRI & SINEXDR & SINELEC & SINAG & HACIN & PISOTI & LOC5000 & INGR \\
\hline Sonora & 5.61 & 28.84 & 32.76 & 9.68 & 8.82 & 26.01 & 17.50 & 25.99 & 52.43 \\
Desierto & 5.73 & 33.85 & 34.03 & 7.72 & 9.32 & 27.21 & 10.50 & 16.72 & 47.91 \\
Río Altar & 6.29 & 42.05 & 42.67 & 17.70 & 14.36 & 26.98 & 15.52 & 100.00 & 47.92 \\
F. Centro & 3.27 & 22.40 & 23.04 & 12.75 & 12.74 & 23.66 & 11.24 & 13.29 & 51.70 \\
F. Norte & 3.11 & 23.91 & 17.15 & 10.40 & 5.16 & 19.25 & 6.58 & 21.58 & 43.94 \\
R. Sonora & 6.78 & 43.02 & 35.87 & 13.81 & 7.41 & 19.68 & 12.48 & 100.00 & 69.07 \\
y S. Miguel & & & & & & & & & \\
Sierra Alta & 5.27 & 39.22 & 32.31 & 10.72 & 4.97 & 16.68 & 6.97 & 100.00 & 54.09 \\
Centro & 7.23 & 49.40 & 46.77 & 14.85 & 12.95 & 19.19 & 16.64 & 100.00 & 63.62 \\
Hermosillo & 3.67 & 21.94 & 21.98 & 6.11 & 4.91 & 22.91 & 14.05 & 6.53 & 41.88 \\
Sierra & 11.39 & 50.21 & 56.83 & 42.21 & 14.51 & 22.05 & 28.28 & 100.00 & 55.87 \\
Guaymas- & 5.98 & 29.14 & 39.76 & 7.13 & 12.75 & 27.72 & 17.73 & 21.64 & 56.39 \\
Empalme & & & & & & & & & \\
Yaqui-Mayo & 6.92 & 30.48 & 39.84 & 9.40 & 8.61 & 29.82 & 25.07 & 25.48 & 61.47 \\
Sierra Baja & 18.92 & 54.59 & 76.34 & 42.49 & 29.97 & 35.74 & 48.04 & 82.50 & 72.71 \\
\hline
\end{tabular}

Fuente: estimaciones propias en base en CONAPO 1993 y XI Censo General de Población y Vivienda 1990.

Otra manera de ver las tendencias es por medio de la gráfica 1. La pendiente más pronunciada de cada curva es la muestra de que el porcentaje de carencia se redujo más rápido. Así las curvas muestran pendiente negativa, excepto la PEA con ingresos menores a dos SM, con pendiente positiva. La mayor inclinación se observa en el tramo 1970-1980, menos en hacinamiento y localidades menores de 5,000 habitantes. 

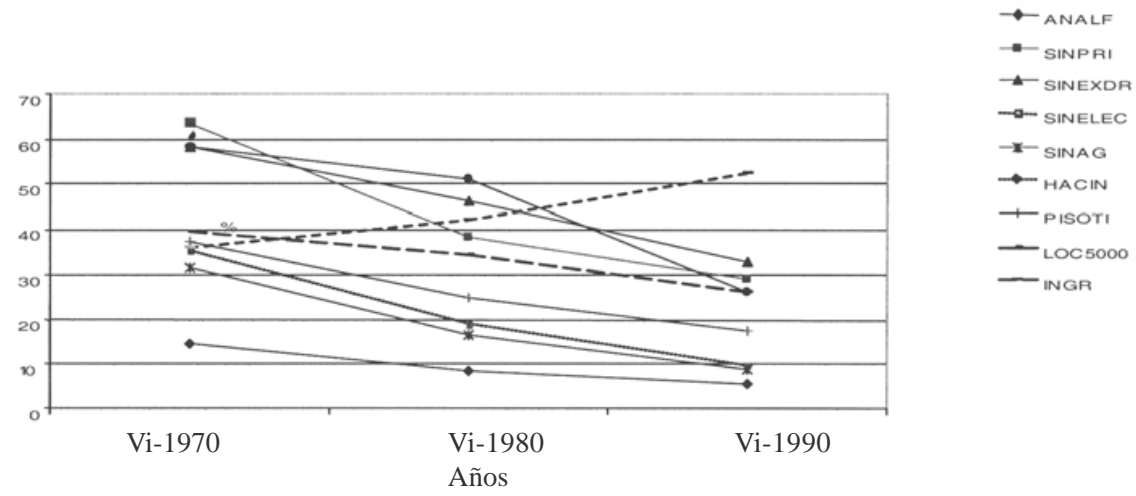

Gráfica 1

Fuente: elaboración propia con base en los cuadros 3 y 4 .

\section{Tendencias de IM de largo plazo}

Los resultados indican (cuadro 5 y gráfica 2) que la marginación en Sonora disminuyó al pasar en 1970 de IM $=-0.876$ a $1980 \mathrm{IM}=-1.275$ más negativo, en tanto que resultó un IM $1990=-1.575$. Disminuyó en ambos periodos, pero los mayores avances se lograron de 1970 a 1980; como puede observarse en la gráfica 2, su pendiente es más pronunciada. La disminución mayor se corresponde con la reducción de las carencias para el periodo. Seguramente los cambios fueron producto de los efectos todavía en los primeros dos años en la década de los ochenta, porque a partir de la crisis económica de 1982, conocida también como crisis de la deuda, hubo en México un abandono de la política social que se reveló en la caída del gasto en los rubros de educación, salud, vivienda e infraestructura urbana, causa de la desaparición del programa COPLAMAR para abatir la marginación.

El análisis regional de la marginación indica que en 1970, ocho de las 12 regiones tenían un IM mayor que el estimado para la entidad, y para 1980, y todavía 1990, seguían siendo prácticamente las mismas ocho regiones las que tenían un IM mayor al del estado, con la salvedad de que no aparece la región Desierto, pero ya se incluye a la región Guaymas-Empalme. 
Cuadro 5

Sonora, tendencias del Iм de largo plazo

\begin{tabular}{lrrr}
\hline & 1970 & 1980 & 1990 \\
\hline Sonora & -0.590 & -1.275 & -1.575 \\
Desierto & -0.802 & -1.260 & -1.685 \\
Río Altar & 0.035 & -0.385 & -1.182 \\
F. Centro & -1.320 & -1.608 & -1.791 \\
F. Norte & -1.283 & -1.609 & -1.990 \\
R. Sonora y S. Miguel & -0.119 & -0.639 & -1.086 \\
Sierra Alta & 0.295 & -0.626 & -1.387 \\
Centro & 0.145 & -0.230 & -0.974 \\
Hermosillo & -1.080 & -1.730 & -1.980 \\
Sierra & 0.429 & -0.093 & -0.627 \\
Guaymas-Empalme & -0.566 & -1.641 & -1.524 \\
Yaqui-Mayo & -0.613 & -1.026 & -1.371 \\
Sierra Baja & 0.084 & 0.039 & -0.015 \\
\hline
\end{tabular}

Fuente: estimaciones propias con base a CONAPO 1990 y censo general de población y vivienda de 1970, 1980 y 1990.

Sonora, tendencia del índice de marginación en la entidad y regional, 1970-1980 y 1980-1990

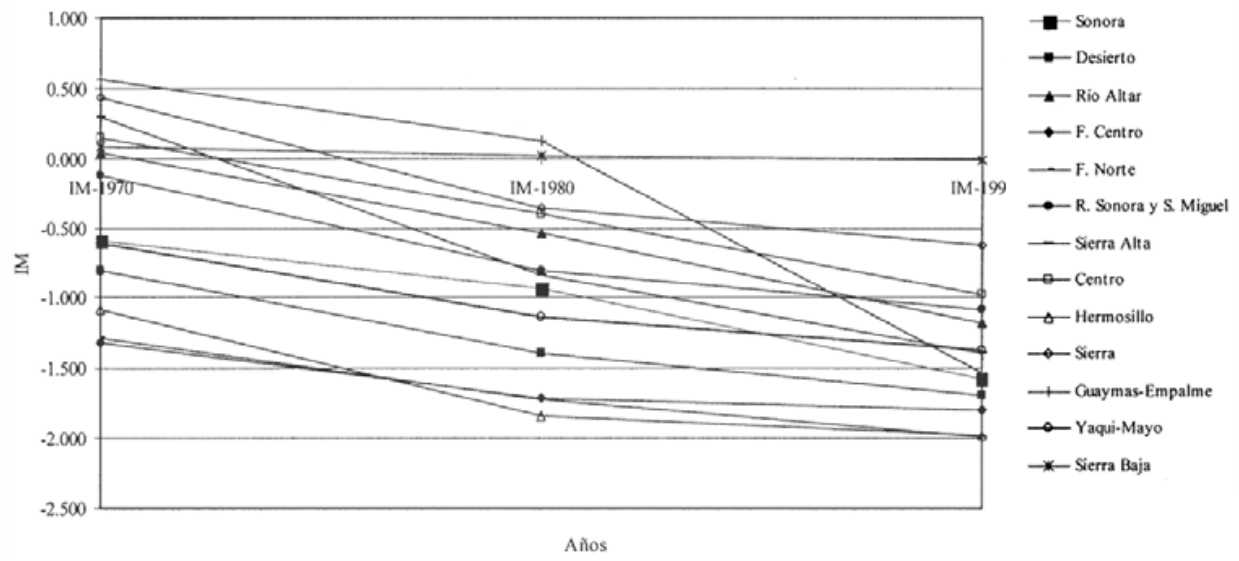

Gráfica 2.

Fuente: elaboración propia con base en el cuadro 5 . 
Cabe destacar el hecho de que las tres regiones con mayor índice de marginación: Sierra Baja, Yaqui-Mayo, y Río Sonora y San Miguel, son predominantemente agrícolas, y las dos primeras son asiento de la mayor cantidad de grupos indígenas del estado; resultaron ser, además, las tres regiones que registraron los mayores niveles de pobreza extrema de Sonora. ${ }^{14}$ Este resultado en las regiones para una entidad confirma lo encontrado en el nivel nacional para los estados de Guerrero, Oaxaca y Chiapas, que observan los más altos índices de marginación, pero también los mayores niveles de pobreza extrema. ${ }^{15}$ Los hallazgos, por lo demás, sirven de sustento a la vieja hipótesis de que uno de los factores que explican el proceso de desarrollo de América Latina es el círculo vicioso entre marginación, pobreza y subdesarrollo.

\section{Sonora y el contexto nacional}

Una forma de evaluar los logros de una entidad es comparándola con los obtenidos por otras, considerando su posición relativa en distintos puntos del tiempo. Al respecto el estudio de CONAPO, basado en los datos de 1990, clasificó a Sonora con marginación baja, solamente superada por Baja California, Nuevo León, Distrito Federal, Chihuahua, Aguascalientes y similar a Jalisco. ${ }^{16} \mathrm{Si}$ bien es cierto que Sonora aparece bien ubicada, es necesario destacar que ha perdido importancia relativa respecto a la que tuvo en otras épocas, cuando las entidades de México se clasificaban por su grado de desarrollo. Así, de acuerdo con estudios realizados por la CEPAL, la antigua Secretaría de Recursos Hidráulicos (parte de lo que ahora es SAGARPa) y de COPLAMAR, en el periodo 1940-1980, era superada por tres entidades: Baja California, Distrito Federal y Nuevo León; por tanto, Sonora se rezagó tres lugares ${ }^{17}$ de 1980 a 1990.

14 Revísese a Camberos y Bracamontes, "Pobreza y desequilibrios regionales", en Carta económica regional, Guadalajara, Universidad de Guadalajara, 2001, pp. 17 24.

15 Genaro Aguilar, La pobreza en México ¿Es inevitable?, México, Porrúa, 2000.

16 CONAPO CNA, Indicadores socioeconómicos e índices de marginación municipal, 1990, México, 1993, pp. 39 40.

17 Consúltese el recuento que hace Mario Camberos, "El Desarrollo Económico y el Deterioro Social de las Regiones de Sonora en el Contexto Nacional", en Rodríguez, Camarena y Serrano, El Desarrollo Regional en México: Antecedentes y Perspectivas, México, UAQ AMECIDER IIEC, 1996, pp. 449 460. 
Esos resultados coinciden también con trabajos en los cuales se muestra un mejoramiento $^{18}$ en Sonora al pasar de un grado de marginación media en 1970 y 1980 a marginación baja en 1990, junto a estados como Baja California Sur, Chihuahua, Colima, Morelos y Tamaulipas.

En estudios más recientes, uno correspondiente a 1995, según el mismo CONAPO, basado en los datos del conteo de población, y referido a los niveles de marginación de los municipios y entidades de México, Sonora permanece en el estrato de marginación baja, superado únicamente por Aguascalientes, Baja California, Coahuila, Distrito Federal y Nuevo León, en los cuales se ubican en el estrato de marginación clasificado como de muy baja, ${ }^{19}$ es decir, Sonora no ha recuperado el lugar perdido.

Por su parte, en el nivel de localidades, CONAPO estimó que para 1995 80\% de la población de Sonora habitaba en municipios cuyo grado de marginación era muy baja; en tanto que, en términos de localidades, 52\% de éstas se ubicaba en los estratos de marginación alta y muy alta, ${ }^{20}$ situación que denota un fuerte abandono de las localidades rurales de Sonora, similar entonces al que se observó en las regiones de México, con mayoría de localidades rurales de los estados del sur y sureste de México. ¿Cómo evolucionaría la marginación de las regiones de Sonora en la última década? ¿Cuáles son las expectativas para el siglo Xxi?

\section{Proyecciones de los indicadores IM 2000}

En esta parte del trabajo elaboramos un ejercicio de simulación de cómo evoluciona el índice de marginación, a partir de los indicadores de carencia (véase cuadro 6 ), las cuales después comparamos con los estimados por el cONAPO en 2000, con el propósito de utilizar el método para establecer tendencias que sirvan de base para el diseño de una política social que prevenga el incremento o el rezago en alguna de las dimensiones de la marginación.

Primero se tomaron los valores esperados de carencia para los nueve indicadores. Luego, con éstos se calcularon los índices de marginación alternos (IN2), para des-

18 García, Flores y Tovar, "México: comportamiento reproductivo y marginación social 1970 1990. Elementos para un diagnóstico geográfico en salud reproductiva”, Salud Pública de México, vol. 37, núm. 4, julio agosto de 1995, pp. 279 287.

19 ConAPO Progresa, Índices de marginación, 1995, México, anexo II, 1998, p. 64.

20 Véase conAPo, Situación demográfica de México, 1997, http:/www.conapo.gob.mx. 
pués obtener los IM correspondientes a cada región. Posteriormente, se estimó una ecuación de regresión y los valores de la pendiente y el término constante, tomando como variable dependiente los IM (1990) de cada una de las regiones y como variable independiente se consideraron los Iм (1970) е Iм (1980); de esta manera, se obtiene una ecuación de regresión para la entidad y para cada región. Tomamos como ejemplo la región desierto, que fue la siguiente:

$$
\operatorname{IM}(1990)=-0.35868-0.62669(\operatorname{IM}(1970))+1.46919(\operatorname{IM}(1980))
$$

Cuadro 6

Sonora, proyección por indicador y región, 2000

\begin{tabular}{lrrrrrrrrr}
\hline & \multirow{2}{*}{ ANALF } & \multirow{2}{*}{ SINPRI } & SINEXDR & SINELEC & SINAG & HACIN & PISOTI & LOC50OO & INGR \\
\hline & & & & & & & & & \\
Sonora & 5.15 & 27.11 & 31.49 & 8.40 & 7.68 & 24.39 & 16.50 & 25.31 & 53.25 \\
Desierto & 5.34 & 32.02 & 32.56 & 6.64 & 8.43 & 25.61 & 9.76 & 16.33 & 48.94 \\
Río altar & 5.96 & 40.56 & 40.91 & 15.59 & 12.57 & 25.87 & 14.23 & 100.00 & 48.22 \\
F. Centro & 2.93 & 20.83 & 21.84 & 12.05 & 12.18 & 22.52 & 10.74 & 12.87 & 52.74 \\
F. Norte & 2.86 & 22.40 & 15.89 & 9.60 & 4.50 & 17.82 & 5.82 & 21.28 & 44.52 \\
R. Sonora & 6.51 & 41.62 & 34.14 & 12.02 & 5.93 & 17.85 & 10.88 & 100.00 & 69.93 \\
y S. Miguel & & & & & & & & & \\
Sierra Alta & 4.95 & 37.66 & 29.90 & 7.53 & 3.06 & 15.05 & 4.38 & 100.00 & 54.26 \\
Centro & 6.92 & 47.89 & 45.24 & 11.90 & 11.14 & 16.83 & 14.80 & 100.00 & 64.58 \\
Hermosillo & 3.28 & 20.30 & 20.76 & 5.33 & 3.96 & 21.51 & 13.47 & 6.10 & 42.41 \\
Sierra & 11.04 & 48.70 & 55.32 & 41.00 & 12.50 & 20.03 & 26.37 & 100.00 & 56.43 \\
Guaymas- & 5.50 & 26.95 & 38.78 & 5.83 & 11.92 & 26.16 & 16.69 & 21.17 & 57.54 \\
Empalme & & & & & & & & & \\
Yaqui-Mayo & 6.36 & 29.02 & 38.84 & 8.07 & 7.30 & 28.06 & 24.02 & 24.64 & 62.62 \\
Sierra & 18.96 & 54.03 & 75.84 & 40.46 & 27.94 & 33.39 & 46.48 & 81.62 & 73.44 \\
\hline
\end{tabular}

Fuente: estimaciones propias con base en CONAPO, 1993 y Censo General de Población y Vivienda, 1970,1980 y 1990.

Con el propósito de obtener las proyecciones en el mediano plazo, debemos calcular los cambios del IM a partir de 1991. Éstos los obtenemos a partir de las diferencias entre los valores encontrados en los periodos 1970-1980 y 1980-1990 para cada región, divididos por diez, que es el número de años que abarca cada lapso. El resultado será la tasa anual de cambio (TAC), la cual se sustituye como variable independiente en las ecuaciones obtenidas para las regiones y la entidad. Así, tendremos la serie de IM en la entidad y regional para 2000. 
De acuerdo con nuestras proyecciones del IM CIAD-2000 de mediano plazo (véase cuadro 7, infra), la marginación de Sonora pudo haber alcanzado un IM $=-1.370$, resultado que ubica como una entidad de marginación baja conforme a la clasificación de CONAPO (cfr. cuadro 1, supra), misma clasificación que obtuvo en 1990.

Del análisis de las regiones encontramos que solamente la Sierra Sur registra un IM positivo, lo cual significa que persiste como la región más marginada, siguiéndoles Río Sonora y San Miguel, así como Yaqui-Mayo. En seguida haremos dos comparaciones conducentes. La primera para probar la magnitud del sesgo de nuestras estimaciones IM CIAD-2000, respecto de los de CONAPO. Después compararemos los resultados con los obtenidos en 1990 para conocer si mejoró la situación de Sonora y sus regiones en la última década.

Cuadro 7

Sonora, índices de marginación IM regionales, proyecciones de mediano plazo

\begin{tabular}{|c|c|c|c|}
\hline Región & IM CONAPO 2000* & 1990 & IM CIAD 2000 \\
\hline Sonora & -0.75590 & -1.575 & -1.370 \\
\hline 1. Desierto & -1.50934 & -1.685 & -1.390 \\
\hline 2. Rio Altar & -1.12822 & -1.182 & -1.430 \\
\hline 3. F. Centro & -1.32689 & -1.791 & -1.391 \\
\hline 4. F. Norte & -1.63529 & -1.990 & -1.679 \\
\hline 5. R. Sonora y S. Miguel & -0.92868 & -1.086 & -1.163 \\
\hline 6. Sierra Alta & -1.07930 & -1.387 & -1.474 \\
\hline 7. Centro & -0.85272 & -0.974 & -1.457 \\
\hline 8. Hermosillo & -1.82000 & -1.980 & -1.403 \\
\hline 9. Sierra & -0.66462 & -0.627 & -1.685 \\
\hline 10. Guaymas-Empalme & -1.45588 & -1.524 & -1.201 \\
\hline 11. Yaqui Mayo & -1.21729 & -1.371 & -1.102 \\
\hline 12. Sierra Baja & 0.15119 & -0.015 & 0.037 \\
\hline
\end{tabular}

*En 1990, CONAPO registra para Sonora IM $=-0.8568<-0.75590$ (2000).

Fuente: CONAPO, Índices de marginación 2000 y estimaciones propias con base en CONAPO 1993 y censos generales de población y vivienda de 1970, 1980 y 1990.

\section{Comparaciones IM CIAD CON IM CONAPO 2000 y 1990}

En lo que se refiere a la comparación con el IM CONAPO 2000 (véase cuadro 7), encontramos similitudes y diferencias que en seguida explicaremos. Respecto a las similitudes: $a$ ) Existe consistencia en las estimaciones de CIAD y CONAPO en tanto que registran los IM negativos para la entidad y 11 regiones; mientras que el índice resultó positivo para la Sierra Baja y de un valor parecido. $b$ ) En nueve de las 12 
regiones, se observa la misma clasificación (supra, cuadro1) con CONAPO ${ }^{21}$ y CIAD, excepto Hermosillo, la Sierra y Centro. c) En ambas estimaciones, la región Río Sonora y San Miguel, le siguen en marginación a la Sierra Baja. Tal vez por eso la población de esas regiones, calificadas de alta marginación, fueron las únicas de Sonora incluidas en el programa Oportunidades-Contigo, ${ }^{22}$ eje de la política social de la actual administración enfocada a las localidades de alta y muy alta marginación.

Al considerar las diferencias encontramos: 1) El índice IM para Sonora es más negativo con las estimaciones CIAD que de CONAPO, si bien es cierto que con ambas la clasificación es de marginación baja. No obstante, pensamos que la estimación del IM CIAD 2000 muestra más congruencia, toda vez que la tendencia desde 1970 es ser más parecidos o bien a tener un valor entre el IM de las regiones Hermosillo y Yaqui-Mayo, que han acumulado cerca de $60 \%$ de la población (véase supra, cuadro 2). En cambio, el valor de CONAPO 2000 está muy por debajo de las dos regiones mencionadas. Es posible que la diferencia resulte del cambio de método que utilizó CONAPO para estimar el IM de las entidades $2000,{ }^{23}$ con respecto de 1990.

2) Las regiones de Hermosillo, Centro y Sierra, muestran diferencias importantes entre ambas estimaciones. La explicación pudiera estar en que Hermosillo rebasó las tendencias, debido a que se puso más atención a las demandas de su población; mientras que pudo existir un abandono en el de las regiones agrícolas del Centro y la Sierra.

\section{¿Mejoró la marginación en la década de los noventa?}

En la última década del siglo xx, los distintos niveles de gobierno de México en turno - conscientes del aumento en la pobreza y la marginación, producto de las crisis económicas y los ajustes para combatirla y que habían alcanzado a grandes capas de la sociedad, particularmente a los grupos más vulnerables de indígenas, los niños y los adultos de la tercera edad, principalmente de las regiones rurales, las madres

21 Nosotros utilizamos la clasificación única de 1993 con fines comparativos del im en el tiempo. CONAPO hizo dos nuevas tablas clasificatorias en 2000, una para las entidades y otra para los municipios. En ambas cambian los valores respecto de 1993. Véase conAPO, Índices de marginación 2000, Anexo C, pp. 191 y 196. Índices de Marginación 2000, Anexo C, pp, 191 y 196.

22 Versión del programa Oportunidades Contigo, Sonora, presentado por la Delegación Regional de SEDESOL Sonora, Hermosillo, junio de 2002.

23 Véase el Anexo C, Índice de marginación 2000. 
solas, los discapacitados - pusieron en práctica, en su momento, dos programas para dar respuestas a las demandas: el Programa Nacional de Solidaridad (PRONASOL) y el Programa de Educación, Salud y Empleo (PROGRESA) por las administraciones salinista y zedillista, respectivamente.

En su momento, el PRONASOL había sido evaluado como medio eficaz para combatir la pobreza; sin embargo, autores reconocidos demuestran que la pobreza extrema creció a lo largo de toda la década de los noventa. ${ }^{24}$ Por su parte, en lo que se refiere a PROGRESA, programa que fue diseñado para atender prioritariamente a las localidades y municipios de alta y muy alta marginación, Boltvinik señalaba, en su momento, las graves limitaciones en cuanto a su cobertura, debido que dejaba fuera un porcentaje de población mayor que la población objetivo, error derivado de las estimaciones realizadas por el mismo CONAPO. ${ }^{25}$ La consecuencia del error es que el programa pudiera no ser suficiente para atender las demandas de la población marginada. ¿Qué ocurrió con la marginación? ¿Fueron suficientes los programas para reducirla? Para dar respuesta a los cuestionamientos en términos de la lógica del desarrollo de este trabajo, compararemos las estimaciones 1990-2000 del propio CONAPO (cuadro 7, supra).

Es indiscutible que en el nivel de la entidad se observó un marcado retroceso durante el periodo, pues, si bien es cierto, mantiene la clasificación de marginación baja, con el índice IM $=-0.75590$ en 2000, se encuentra más cerca de la media, mientras que en 1990 con un $\mathrm{IM}=-1.575$, se ubicaba más cerca de la marginación baja.

La mayoría de las regiones también sufrieron retrocesos o mantuvieron el mismo nivel de marginación. Entre las primeras se encuentran las dos regiones fronterizas, la Sierra Alta, Hermosillo, Yaqui-Mayo y Sierra Baja. Ninguna de las regiones de Sonora mejoró su posición en 2000 respecto de 1990. Esos hallazgos, salvo errores posibles en las estimaciones del propio CONAPO, nos llevan a pensar que los programas PRONASOL y PROGRESA, si no pudiera calificárseles como un fracaso total, sí lo son en el sentido que no han cumplido con el objetivo para el cual fueron

24 Véase al respecto a Boltvinik y Hernández, Pobreza y distribución del ingreso en México, Siglo xxi, México 1999, y Aguilar, Desigualdad y pobreza en México ¿son inevitables?, Porrúa, UNAM, IIEc, IPN, CIECAS, México, 2000, pp. 86 87.

25 Boltvinik, "Los excluidos del Progresa", en Hojarasca, suplemento mensual, La Jornada, 4 de septiembre de 1999. 
diseñados: reducir la marginación y la pobreza extrema en municipios y localidades de alta marginación.

Una muestra del fracaso general de la política social en México, que confirma los hallazgos en la fronteriza Sonora, la encontramos en las estimaciones de CONAPO, ${ }^{26}$ en el cual se muestra que del total de 2, 443 municipios existentes en 2000, 905 eran de alta marginación, comparado con 842 en 1990; mientras que los clasificados como de muy alta marginación fueron 386 y 341 respectivamente. Eso significa que en México la marginación en la última década del siglo xx se incrementó $7.5 \%$ y $13 \%$.

Existen diversas explicaciones del fracaso de la política social. Primera. La relación que se establece no es siempre en los mejores términos entre los agentes del cambio, y los hogares o individuos que son el objeto del cambio, quienes desconocen, en la mayoría de las ocasiones las políticas y programas sociales que desarrollarán en su comunidad y en el hogar.

Segunda. La falta de una evaluación adecuada de los mismos "al tomar como patrón de medición indicadores de ejecución presupuestaria, cobertura, cobertura y costos mínimos de prestación de servicios [...] Casi ninguno da cuenta del impacto logrado por las políticas y programas en función de los objetivos sociales que las justifican". ${ }^{27}$

Tercera. La evaluación de los programas sociales, incluido OPORTUNIDADES-CONTIGO, realizada por los propios beneficiarios, da cuenta que menos de una tercera parte de la población objetivo recibió alguno de sus beneficios. ${ }^{28}$ Es excluyente, como lo fue PROGRESA, según lo anotaba Boltvinik (supra: 24); por tanto, insuficientes para reducir la marginación.

26 CONAPO, Índices de marginación 2000, cap. 3, cuadro 25, p. 24 y cap. 2, p. 21.

27 Para un análisis metodólogico de la evaluación de los programas sociales, véase González, "La evaluación de los programas sociales aspectos conceptuales y prácticos", Economía Informa, núm. 303, diciembre de 2002, pp. 22 27.

28 Hernández y Lilia del Razo, Lo que dicen los pobres: evaluación del impacto de los programas sociales sobre la percepción delos beneficiarios, México, SEDESOL, 2004. 


\section{Conclusiones}

Es fundamental estimar los niveles de marginación toda vez que junto con los de pobreza constituyen las dos mejores medidas para evaluar las políticas sociales cuyo objetivo es abatirlas. Por ello, con base en el método de CONAPO, a partir de los segundos IM 1990, apoyados en regresiones lineales para determinar las tendencias, encontramos para Sonora en 1970 un Iм $=(-0.876)$, que la clasificaría como de marginación baja; mientras que el de 1980 Iм $=(-1.275)$ y el de 1990 $\mathrm{IM}=(-1.575)$ le colocaban todavía en el estrato de marginación baja.

En lo que respecta a las proyecciones de mediano plazo, en 2000 la entidad alcanzó un índice de marginación IM $=(-1.370)$ que la clasificaría como de marginación baja, similar a la del CONAPO, si bien más cerca de la marginación media, señal de retroceso en la última década del siglo xx, como ocurrió con México.

También es necesario considerar las proyecciones de indicadores y del IM para conocer las tendencias y establecer metas de logros para modificarlas, cuando lleven a incrementar una o varias de las carencias que pusiera en riesgo la de por sí precaria estabilidad política y social de las regiones.

Para terminar, es prudente resaltar que una condición necesaria para no sufrir retrocesos en el combate a la marginación es el establecimiento de una política social cuya realización no dependa del gobierno en turno sino que los recursos presupuestarios estén garantizados en términos reales y la evaluación de los programas y acciones que la componen se realicen más in situ y menos en gabinete como un ejercicio independiente del Ejecutivo, todo ello con el objetivo de reducir la marginación en la presente década.

\section{Bibliografía}

Aguilar, Genaro, La desigualdad y la pobreza en México ¿son inevitables?, Porrúa, UNAM, IIEC, IPN, CIECAS, México, 2000.

Ayala, B. Rosa, Distribución espacial de la población de alta y muy alta marginación por altitud en México, SEDESOL-Progresa, Http:/www.sedesol.gob.mx, 1999.

Boltvinik, K. Julio, y E. Hernández L., Pobreza $y$ distribución del ingreso en México, México, Siglo xxi, 1999.

___ "Los excluidos del Progresa", Hojarasca, La Jornada, año 15, 4 de septiembre, Suplemento mensual, 1999.
,Pobreza y estratificación social en México, México, INEGI-COLMEX, IIS-UNAM, 1994.

Camberos M. y J. Bracamontes, "Pobreza y desequilibrios regionales", Carta económica regional, Guadalajara, México, UdeG, julioseptiembre, de 2001, pp. 17-44.

y Jaime Arturo Yánez V., "La pobreza regional en los noventa: estrategia, medidas para combatirla y costos de financiamiento", Economía y sociedad, año 9, núm. 13, Morelia, México, Facultad de Economía, Universidad Michoacana de San Nicolás de Hidalgo, enero-junio 2004, pp. 37-51. 
- "El desarrollo económico y el deterioro social de las regiones de Sonora en el contexto nacional", en Salvador Rodríguez, Margarita Camarena y Jorge Serrano M., El Desarrollo regional en México: antecedentes y perspectivas, México, UAQ-AMECIDER-IIEc, pp. 449-460, 1996.

CONAPO, Índices de marginación 2000, México, 2002.

, La población de México en el nuevo siglo, México, 2001.

— L L L situación demográfica de México: La marginación de las localidades de México, 1995. http:/www.conapo.gob.mx, 1997.

— PROGRESA, Índices de marginación 1995, anexo II, México, 1998.

CONAPO-CNA, Indicadores socioeconómicos e indices de marginación municipal, 1990, México, 1993.

Comisión de Desarrollo Social, H. Congreso de la Unión, Paquete legislativo de desarrollo social, iniciativas presentadas durante la LXIII Legislatura, México, mimeo, 2002.

COPlamar, Geografía de la marginación, México, S. xxi, 1983.

Cordera, C. Rolando y A. Palacios, "La cohesión social en tiempos mutantes: algunos desafíos", Economía Informa, núm. 303, México, unam, enero de 2002, pp. 5-21.

Cortés, F. "Consideraciones sobre la marginación, la marginalidad, marginalidad económica y exclusión social", Papeles de población, núm. 47, UAEM, 2006, pp. 71-84.

Cruz E., R. Pérez y Sergio de la Vega, "Geografía de la Marginación y desarrollo de Progresa", en Evaluación de resultados de Progresa. Primeros avances, 1999, http:/www.sedesol.gob.mx, 1999, pp. 3356.
Díaz Polanco, H. y Michel Díaz, J., Indigenismo, modernización y marginalidad, México, Juan Pablos, 1981.

García C., M. Flores y V. Tovar, "México: Comportamiento reproductivo y marginación social 1970-1990. Elementos para un diagnóstico geográfico en salud reproductiva", Salud pública de México, México, vol. 37, núm. 4, julio-agosto de 1995, pp. 279-287.

González S. Gloria, "Algunos indicadores y comentarios generales sobre el rezago educativo de Chiapas", Momento económico, núm. 72, México, UNAM, marzo-abril de 1994, pp. 7-10.

González T. Enrique, "La evaluación de los programas sociales aspectos conceptuales y prácticos", Economía Informa, núm. 303, México, unAm, enero de 2002, pp. 22-27.

Hernández G., G. Merino y E. Rascón, Desarrollo social en México, México, SEDESOL, 2003.

Hernández, G. y Lilia M., del Razo, Lo que dicen los pobres: Evaluación del impacto de los programas sociales sobre la percepción de los beneficiarios, México, SEDESOL, 2004.

INEGI, XII Censo General de Población y Vivienda. Sonora, México, 2000.

INEGI, XII censo general de población y vivienda. Sonora, México, 1992.

SEDESol, Delegación regional de Sonora, Programa Oportunidades-Contigo, Sonora, Hermosillo, 2002.

spP-Gobierno del Estado de Sonora, Plan Estatal de Desarrollo 1980-1985, Hermosillo, México, 1980.

SPP-INEGI, x Censo General de Población y Vivienda, Sonora, México, 1984.

SIC-DGE, IX Censo de Población, Sonora, México, 1974.

Trejo, Guillermo y Claudio Jones, Contra la pobreza, México, Cal y Arena, 1993. 


\section{VOICES of Mexico}

CISAN • UNAM

U.S. Midterm Elections

Views from Mexico

Articles by

José Luis Valdés-Ugalde,

Silvia Nuñez, Paz Consuelo

Márquez-Padilla

and Alejandro Anaya

President Calderón and the United States

J. Jesús Esquivel

Governability and

Multilateral Bodies

Antonio Ortiz Mena López Negrete

Mexico's Economic

Safeguards

Pablo Ruiz Nápoles

An Interview with

Sculptor Federico Silva

Remembering Salvador Elizondo's Literature

Real de Catorce and Xilitla

The Enchantment of

San Luis Potosí

\section{.}

